\title{
Intrinsic Optical Fiber Sensor
}

\author{
Sylvain Lecler and Patrick Meyrueis \\ Strasbourg University \\ France
}

\section{Introduction}

The use of more and more complex systems in transportation, bio medical devices, defence, production, systems, etc., that are more and more automated, having to comply with many norms related to low energy consumption, miniaturization, insensivity to modifications of the surrounding conditions, security, etc. induce the need of new sensors that have to be reliable, low cost, easy to produce, if possible without clean rooms, avoiding too demanding calibration process and using no poisonous components or materials.

Optical fiber sensors were initially elaborated to validate some light propagation modelling in optical fiber. Initially, they were fragile, only performing in laboratory conditions and, most of the time, they were potentially more expensive to realize than other technology sensors, and they had a reliability factor that was not excellent.

But with the improvement of knowledge in guided optic modelling, lowering of tolerance of manufacturing in guided optics components, improvement of optical fiber connection technology, new way to extract physics modulation parameters from light modulation in polarization, phase, frequency, intensity have appeared.

It was thus discovered that optical fiber sensors have a very wide range of effective applications and that the packaging of optical fiber sensors for industry fit more and more the need for quality and performance that is the one of modern products.

In fact, two kinds of optical fiber sensors arised in the market: the extrinsic and the intrinsic optical fiber sensors. In the first case, the transducer, that is not an optical fiber, is modulating the light, out of a physics phenomena: for instance a vibration, and this modulated light is then sent inside the fiber where it will propagate up to a device converting, at the output of the fiber, the modulated light into a modulated electronic signal. Since only light intensity modulation can be read by these devices it is necessary to convert a light modulation that is not intensity into light intensity modulations. Many optical fiber designs allow this task. The one that will be selected will fit the application needs. Extrinsic sensors are generally not dedicated to high level measurements, but they are convenient for applications where low resolution and low cost are requested.

Intrinsic optical fiber sensors are sensors that have a totally different design. The conversion of the modulation of a physic parameter is done by a portion of an optical fiber. These sensors are much more difficult to elaborate, but they have the best performances. The first ones to be developed for mass production were the Sagnac gyro-sensors: they are based on 
the speed limit of light in a media with a relativity effect and a close optical loop in which the photons are circulating in opposite guided directions. Their interferences give, with simple relation the output signal that is a rotational acceleration. A gyroscope is thus constituted. Such gyros are intensively used now for transportation navigation, including satellite.

We can also classify the optical fiber sensors by the kind of optical fiber that constitutes them. So we will have multimode optical fiber sensor, and monomode optical fiber sensor. The difference is lying on the performances of the fiber used: multimode fibers have a low band pass but they are easy to assemble, it is the contrary for the monomode device. The effect of perturbation of their surroundings to the light propagating in the fiber is easy to model with a monomode device and more difficult with the multimode fiber. So the initial trend was to use monomode fibers for sensing, but more and more smart uses of multimode fibers are implemented in sensing devices because of their advantages.

The development of multimode fiber sensors induces the use of polymer fiber, much cheaper, and that can be cut and assembled in an economic way, but they cannot assume any kind of measurements. The most rewarding use of polymer fiber in sensing was done for measuring shock and vibration.

The more recent evolution of optical fiber sensing is toward integrated optics. The optical circuit allowing the physics phenomena detection through guided wave is implemented in a chip that will incorporate sensitive optical waveguide. This allows the realization for instance of biochips for sensing biological molecule by constituting some kind of a "lab on a chip".

The very last technical contribution of innovation to optical fiber sensors are plasmonic effects and photonic crystal fiber. They allow to do measurement that were out of reach before like hydrogen concentration, by using new effects connected to the specific way light is propagating in this case.

So it can be said that optical fibers sensing has matured but is still in a development process: even if effective products are more located in the defence, ecology or biomedical domain plenty of other commercial products are now proposed for planes and automotives. These devices are also named because they are a mixing of micro optics, mechanics, electronics, micro optomecatronic devices.

As it is scheduled in the Photonics 21 prepared for the European Union forecast, the future will see many new technologies of this kind. Photonics being an enabling technology acknowledged by the European Union as one of the 5 KET (Key enabling technology). This enabling work will be as relevant in the field of optical fiber sensing than in other areas of photonics.

\section{Intrinsic optical fiber sensors: A matured technology}

\subsection{Extrinsic and intrinsic optical fiber sensors}

Initially in the seventies, the aim of optical fibers and components was to transmit light without perturbation. The fiber was used as a connexion to transport information. The possible light perturbations were only studied in order to be minimized. In contrary, an 
optical fiber sensor is a system where information is created in the optical path way due to an interaction with a physical phenomenon to be measured and before its transmission to a detector using one or several optical fibers. If the optical fiber is only used to transmit the light, we talk about an extrinsic optical fiber sensor. If the modulation of one of the properties of light takes place in the optical fiber due to the physical phenomenon to measure, we talk about an intrinsic optical fiber sensor [Ferretti, 1996].

\section{Sensing principle}

A change in pressure, temperature, curvature and so on will modify such properties of the optical fiber propagating light as its phase, polarization, amplitude or spectre.

For each of these physical parameters corresponds a family of intrinsic optical fiber sensors that will be described.

\subsection{Optical fiber sensors tools improvements}

Any optical fiber sensor is composed of source(s), optical fiber(s), transducer(s), detector(s) and a signal analyzing system. The recent advances in photonics, nanotechnology and telecommunications have increased the number of available technologies of all this components, allowing new innovative sensors.

\section{Available sources}

There is always more available sources:

- The incoherent Light Emitted Diode (LED): low cost and available in always more spectral range from UV to infrared using new semiconductor.

- The super-luminescent diode (SLD): more powerful than a classical LED. More generally, a lot of progresses have allowed increasing the LED output power.

- The coherent Laser Diode (LD): available in always more spectral range from UV to infrared. Their cost has decreased. They can be pigtailed (Fig.1) and power controlled.

- The Vertical-Cavity Surface-Emitting Laser (VCSEL), more powerful than classical LD but less coherent. They are often multimode.

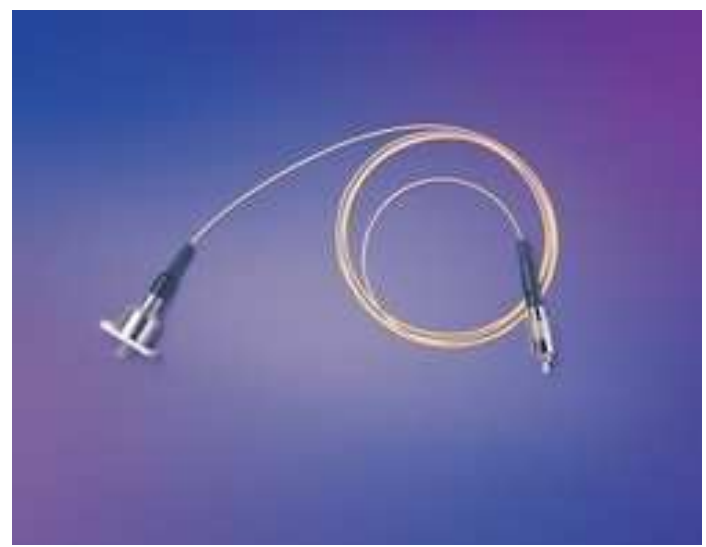

Fig. 1. Example of pigtailed Laser Diode. 


\section{Always more optical fibers}

Due to telecom applications, the classical step index fibers in silica are very cheap $(\sim 0.10 \$ / \mathrm{m})$. However beyond these specific applications, a large number of new fibers is nowadays available with different materials and geometries. Some of them can be customermade. Among the available optical fibers, we can distinguish:

- The monomode and multimodes optical fibers, with core diameter from 5 microns to the millimetre.

- $\quad$ The polarization maintaining fibers (generally with an elliptical core).

- The step index fibers and the graded index ones with several index profiles.

- The fibers whose core and cladding are in silicate or polymer (plastic). A lot of improvements have been achieved concerning plastic fiber [Peters, 2011]: loss decrease, temperature resistance, homogeneity, etc.

- The silica fibers with several possible doping (germanium, fluorine, alumina, erbium, etc.).

- The micro-structured fiber in the radial direction such as the photonic crystal fibers.

- The Bragg grating fiber (with a periodic modulation of the refractive index in the longitudinal direction).

Several coatings can be used. Depending on applications, they can allow the sensitization of a fiber to a physical phenomenon to be measured or on the contrary its neutralization. Some possible coatings:

- $\quad$ Plastic (polymer, PPMA, etc.);

- Metallic (Silver, gold, palladium, copper, etc.);

- Magnetostrictive ;

- Electrostrictive ;

- Etc.

\section{Transducers}

The transducer is the main part of the sensor. It's the sensitized part, the location where the system has been designed in order to allow an external physical phenomenon to interact with the light propagating in the fiber. The transducer has to be selective, that is to be sensitive to phenomena that have to be measured without being sensitive to others.

In an intrinsic optical fiber sensor, the transducer converts the physical value to be measured into an optical information. It can be a thermo-optic, elasto-optic, magneto-optic, electo-optic, acousto-optic one, etc.

The optical fiber is generally weakly sensitive to external physical parameters (temperature, pressure, vibration, etc.). Generally, a perturbation has to be applied to increase its sensitivity. Example of perturbations:

- Micro-bending;

- Thermo-sensitive coating;

- Mechanical strengths;

- Curvatures. 


\section{Available detectors}

Depending of the optical parameter that has been modified by the physical phenomenon to be measured, several detectors can be used. The detector is not only a light sensitive area but often a smart micro system:

- Photodiode for amplitude (intensity) monitoring; in silica for visible light detection (Fig.2) and in GaAs for infrared radiation. It is a cheap component. An amplification and transimpedance circuit can be integrated in the component.

- Polarizer, wave-plates and photodiode for polarized state determination. These components can be pigtailed or achieved in micro-optic. Components using liquid crystal or electro-optic components can be dynamically controlled. Alignment of these components is an issue. Their prices decrease but are still expensive.

- Spectrometers for spectral analysis from 0.1 to $5 \mathrm{~nm}$ resolution, from small to large range, with punctual, linear or two-dimensional detectors. In some case the use of a monochromater can be replaced by a wavelength-scanning laser. Large improvements have been achieved in the packaging of spectrometer allowing industrial applications. These devices are still quite expensive.

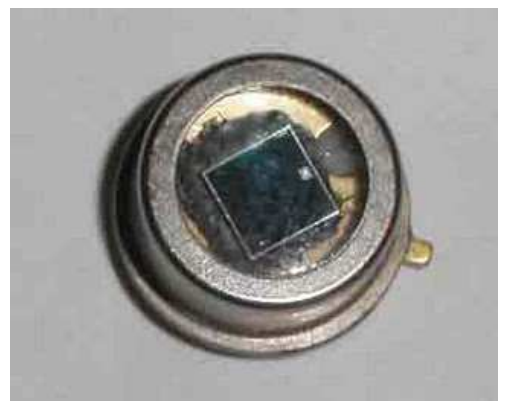

Fig. 2. A photodiode.

\subsection{Known advantages of optical fiber sensors}

Compared to electric or mechanical sensors, the optical fiber ones have several advantages:

- Their high electromagnetic compatibility, because of the superposition principle in the Maxwell equation. [Grattan et al., 2000 | Lee, 2003].

- The possibility to achieve extended and distributed sensors allowing in situ measurements.

- The compatibility to explosive environments. Generally, no current is needed in the sensitized part.

- The low cost components due to Telecom applications.

- The high integration possibility.

- $\quad$ The long time duration of optical fiber and components.

- The large available dynamic.

- The high sensitivity.

- No moving mechanical part. Useful when the sensor is submitted to high accelerations. 


\subsection{Methods to sensitize an optical fiber}

Historically, optical fibers were achieved in order to carry out long distance propagation and some of their main issues were to minimize losses and possible disturbances: regularity of the geometry, purity of the material, protection of the external jacket. Today $0.2 \mathrm{~dB} / \mathrm{km}$ is achieved for long distance telecom connexions (monomode fibers).

In contrast to optical fiber sensor applications, we want to improve the optical fiber sensibility to an external physical parameter. This can be done mainly in three ways:

- Apply geometric perturbations: curvatures, micro-bending, non axis-symmetric deformations, etc.

- Choose specific sensitive materials: materials with magneto-optic, electro-optic, thermooptic, elasto-optic properties; materials able to adsorb other ones;

- Remove the external jacket or replace it by a system able to enhance the external physical parameter effect on the fiber.

\section{Monomode optical fiber intrinsic sensing: The principles}

An optical fiber is monomode when only the fundamental mode is propagative. It is the case when the normalized frequency $\mathrm{V}<2.405$. The normalized frequency $V$ of an optical fiber of radius $a$, numerical aperture $N A$, with a source having a free space wavelength $\lambda_{\mathrm{o}}$ is:

$$
V=\frac{2 \pi}{\lambda_{0}} a \mathrm{NA}
$$

If the refractive index of the core and respectively the optical cladding is $n_{c}$ and respectively $n_{g}$, the numerical aperture of the fiber is:

$$
\mathrm{NA}=\sqrt{n_{c}^{2}-n_{g}^{2}}
$$

Example: the monomode silica/silica fibers are the optical fibers used for long distance communication applications with near infrared sources $\left(\lambda_{0}=1.3 \mu \mathrm{m}, \lambda_{o}=1.5 \mu \mathrm{m}\right)$. The core is generally doped with germanium $n_{c}=1.465, n_{g}=1.455$, their radius is smaller than 10 microns.

In fact a monomode fiber has 4 degenerate modes, that means 4 different modes with the same propagation constant: a TE and TM fundamental modes in two propagating directions. Perturbations can break this degeneration.

Three main families of optical sensors can be achieved using monomode optical fibers: the sensors where polarization or phase or reflected wavelength is modulated by an external physical parameter. For these three families, a single initial possible propagation constant is needed.

\subsection{Polarization modulation}

In this case, the physical phenomenon to be measured modifies the polarization of light propagating in a monomode optical fiber. The fiber has to be monomode because of the depolarization process taking place in the multimode optical fiber. 


\section{Polarization}

The polarization describes the evolution of the electric field direction during time and especially its regularity.
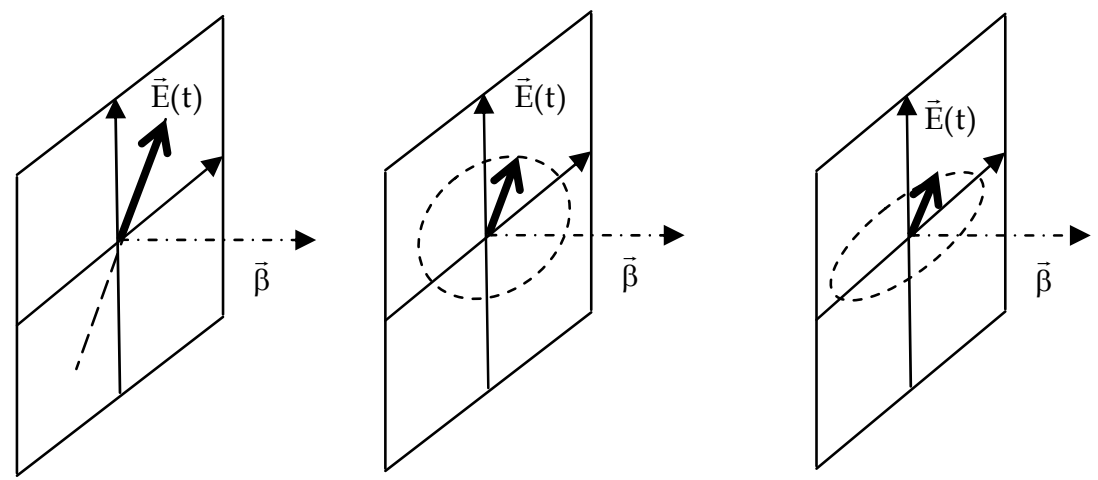

Fig. 3. Linear, circular and elliptical state of polarization.

The polarization is defined only for transverse waves, that is when the electric field or the magnetic one is orthogonal to the propagation vector $\boldsymbol{\beta}$. The classical defined states of polarization are the linear, circular or elliptical polarization following the shape described in the transverse plane by the electric field vector during time (see figure 3 ).

More generally, a state of polarization can be described using the Stokes elements. If $x$ and $y$ are two orthogonal directions in the transverse plane, the Stokes elements are defined as:

$$
S=\left(\begin{array}{c}
\mathrm{S}_{0} \\
\mathrm{~S}_{1} \\
\mathrm{~S}_{2} \\
\mathrm{~S}_{3}
\end{array}\right)=\left(\begin{array}{c}
<\mathrm{E}_{\mathrm{x}} \mathrm{E}_{\mathrm{x}}^{*}>+<\mathrm{E}_{\mathrm{y}} \mathrm{E}_{\mathrm{y}}^{*}> \\
<\mathrm{E}_{\mathrm{x}} \mathrm{E}_{\mathrm{x}}^{*}>-<\mathrm{E}_{\mathrm{y}} \mathrm{E}_{\mathrm{y}}^{*}> \\
<\mathrm{E}_{\mathrm{x}} \mathrm{E}_{\mathrm{y}}^{*}>+<\mathrm{E}_{\mathrm{y}} \mathrm{E}_{\mathrm{x}}^{*}> \\
i\left(<\mathrm{E}_{\mathrm{y}} \mathrm{E}_{\mathrm{x}}^{*}>-<\mathrm{E}_{\mathrm{x}} \mathrm{E}_{\mathrm{y}}^{*}>\right)
\end{array}\right)
$$

Where $\left\langle A>\right.$ represents the time mean value of $A$ and $A^{*}$ the conjugate one. Generally, the Stokes elements are normalized by $\mathrm{S}_{0}$, the total radiated power. The Stokes elements are intensity terms which have the advantage to be deduced by means of measurements; however, they do not constitute a vector space, because the sum of two Stokes elements does not necessary correspond to a physical state of polarization. The degree of polarization is given by:

$$
p=\frac{1}{S_{0}} \sqrt{S_{1}^{2}+S_{2}^{2}+S_{3}^{2}}
$$

$p$ cannot be larger than 1 and smaller than $0 . p=1$ corresponds to a perfectly polarized wave and $p=0$ to a non polarized wave. The classical states of polarization are:

$\mathrm{S}=\left(\begin{array}{llll}1 & 1 & 0 & 0\end{array}\right)$ linear polarization parallel to $\mathrm{x}$; 
$S=\left(\begin{array}{llll}1 & -1 & 0 & 0\end{array}\right)$ linear polarization parallel to $y$;

$\mathrm{S}=\left(\begin{array}{llll}1 & 0 & 1 & 0\end{array}\right)$ linear polarization at $45^{\circ}$ of direction $\mathrm{x}$ and $\mathrm{y}$;

$S=\left(\begin{array}{llll}1 & 0 & -1 & 0\end{array}\right)$ linear polarization at $-45^{\circ}$ of direction $x$ and at $-135^{\circ}$ of $y$;

$S=\left(\begin{array}{llll}1 & 0 & 0 & 1\end{array}\right)$ circular right polarization ;

$\mathrm{S}=\left(\begin{array}{llll}1 & 0 & 0 & -1\end{array}\right)$ circular left polarization.

All the physical Stokes elements can be described in the Poincare sphere or bead (Fig.4).

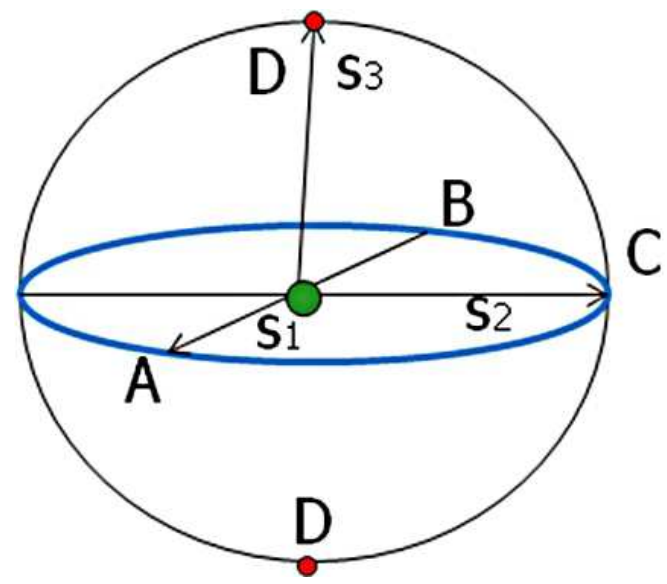

Fig. 4. Poincare sphere describes all the physical possible states of polarization

The perfectly polarized waves are on the sphere surface, the non polarized ones are in the centre, in between we find the partially polarized wave $(0<p<1)$. The poles correspond to the two circular polarization states and the equator describes the linear polarization states. The others points on the surface correspond to elliptical polarization states.

\section{Mueller matrix}

If the initial polarization state is described by a Stokes element, its evolution can be described by a $4 \times 4$ matrix called the Mueller matrix.

\section{Sensor structure and principle}

In a polarization modulated optical fiber sensor, the source has to be monochromatic, polarized but not necessary coherent. The change of polarization can be explained by a change of birefringence in the optical fiber due to the physical phenomenon to be measured. The polarization can rotate, change of state or both.

The detector is generally constituted of a polarizer and a photodiode. The change in the polarization state produces an intensity modulation measured by the photodetector after the polarizer. The calibration curve links the modulation intensity ranging from Imin to Imax and the physical phenomenon to be measured.

\section{Birefringence}

The birefringence is an anisotropy of the optical properties. In the main case, this anisotropy will bring about a change in the polarization state. 
Depending on the polarization eigen mode, the birefringence can be linear or circular. The eigen polarization modes are the modes whose polarization is conserved after propagation in the medium. In the case of the circular birefringence the eigen modes are the circular left and right polarizations. In the case of the linear birefringence, the eigen modes are two orthogonal linear polarizations parallel to two axes called the extraordinary and ordinary axes. These polarizations have different refractive indexes written $n_{e}$ and $n_{0}$. This birefringence can be uniaxial if the refractive index is $n_{e}$ or $n_{0}$ in the third direction or else biaxial.

The birefringence breaks the degeneration of the two modes TE and TM and two propagation constants have to be considered:

$$
\Delta \beta=\left|\beta_{e}-\beta_{o}\right|=\frac{2 \pi}{\lambda_{0}}\left|n_{e}-n_{o}\right|=\frac{2 \pi}{\lambda_{0}} \Delta n
$$

After propagation in a birefringent optical fiber of length $l$, a phase shift $\Delta \Phi$ appears between the two modes that changes the polarization states.

$$
\Delta \Phi=\frac{2 \pi}{\lambda_{0}} l \Delta n=\Delta \beta . l
$$

In a monomode optical fiber the birefringence is due to an asymmetrical geometry, if the core has an elliptical shape for example, or an anisotropy of the medium itself. Two kinds of birefringence can be distinguished: the intrinsic birefringence due to the process of fabrication and the natural defaults of the fiber and the extrinsic one due to external perturbations. The intrinsic birefringence is characterized by the polarisation beating length, which is the smallest length of propagation in order to find again the same polarization as the source. In a classical silica monomode optical fiber used for telecom application the polarization beating length is around $1.2 \mathrm{~m}$.

An optical fiber sensor by polarization modulation uses the extrinsic birefringence. Several external perturbations can create an extrinsic birefringence. We will present several of them.

\section{Curvature}

In an optical fiber curvature, two different phenomena may result in a birefringence, the geometry modification and the elasto-optic effect. The elasto-optic effect is a change in refractive index due to a mechanical stress in the medium. An anisotropic stress may achieve optical anisotropy.

The birefringence due to an optical fiber curvature as described in figure 5 is given by:

$$
\Delta n=\frac{1}{4} n_{c}^{3}\left(p_{11}-p_{12}\right)(1+v) \frac{r^{2}}{R^{2}}
$$

Where :

$R$ is the curvature radius,

$r$ is the optical fiber core radius,

$n_{c}$ is the core refractive index,

$P 11-P 12$ are the elasto-optic coefficients $(P 11=0,121$ and $P 12=0,27$ for silica),

$v$ is the Poisson coefficient (silica: $v=0,17$ ). 
Fiber radius
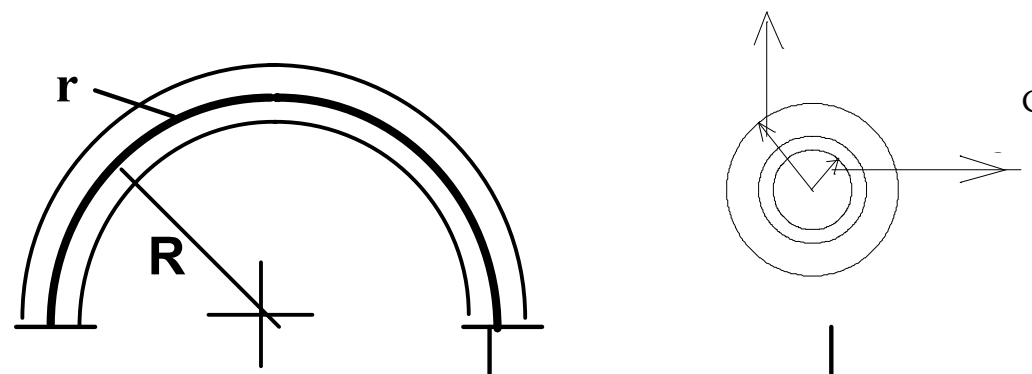

Core radius

Fig. 5. Optical fiber curved with a radius R, without additional stress

The fast birefringence axis (corresponding to the lowest refractive index) is in the curvature plane and the slow one (corresponding to the highest refractive index) is parallel to the curvature axis.

One of the applications is the Lefèvre loop. In this case, an optical fiber loop is made in order to achieve the equivalent of a half or quart wavelength plate.

\section{Compression}

A directional force applied on the optical fiber as described in figure 6 brings about stresses in the optical fiber and, therefore, a linear optical birefringence:

$$
\Delta n(F)=2 n_{c}^{3}\left(P_{11}-P_{12}\right)(1+v) F /(\pi r E)
$$

Where:

$-F$ is the force by fiber length unit $L$,

$-E$ is the Young modulus of the fiber.

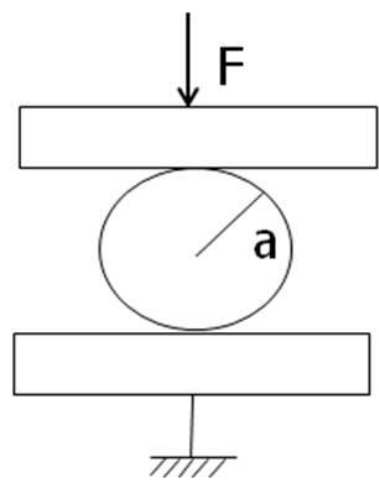

Fig. 6. Directional force applied on an optical fiber.

The fast polarization axis of birefringence is orthogonal to the force $\mathrm{F}$.

Therefore, the phase shift between the two linear polarized modes is: 


$$
\Phi_{x-y}(F)=4 n_{c}^{3}\left(P_{11}-P_{12}\right)(1+v) F L /(\lambda r E)
$$

\section{Torsion}

When an optical fiber is twisted, a circular birefringence appears. The difference of propagation constant between the two circular polarized modes left and right $\Delta \beta$ is proportional to the twist ratio $\tau$ expressed in $\mathrm{rad} / \mathrm{m}$ :

$$
\Delta \beta=g \tau
$$

$g$ is a adimensional constant depending on the medium:

$$
g=n^{2}\left(P_{11}-P_{12}\right) / 2
$$

This birefringence makes a linear polarization to rotate at a ratio $g \tau / 2(\mathrm{rad} / \mathrm{m})$.

\section{Electric and magnetic field}

A magnetic field (static or low frequency) may also be able to achieve a circular birefringence because of the Faraday effect. The rotation angle of an incident linear polarization a will be:

$$
\alpha=v L B
$$

Where:

$v$ is the Verdet constant.

$L$ is the length of fiber where the magnetic field is applied.

$B$ is the magnetic field component parallel to the optical fiber axis.

The electric field due to the Pockels in a crystal can also make change the polarization by a linear birefringence and therefore be detectable. The same method can be used to measure voltage: a voltage $V$ between two conductive plates separated of a distance $d$ achieve an electric field $V / d$ orthogonal to the plates.

\subsection{Phase modulation}

In this case, the external physical phenomenon to be measured modifies the phase shift between two coherent propagating beams having different paths.

\section{Mach-Zehnder}

Most phase modulated optical fiber sensors are interferometers. Using a coupler, the light source is guided through two optical fibers: one is the reference arm, the other one is the measurement arm. Using a second coupler the two beams are again put together (Fig.7). [Yuan et al., 2000 | Ghorai et al., 2005].

The fiber has to be monomode, otherwise each mode would have a different phase shift linked to its eigen propagation constant. The coherence length of the light source has to be larger than the maximum possible difference of the optical path between the two arms.

The phase shift will be linked to a refractive index change in the measurement arm. This refractive index modulation can take place in the optical fiber core or cladding due to a 


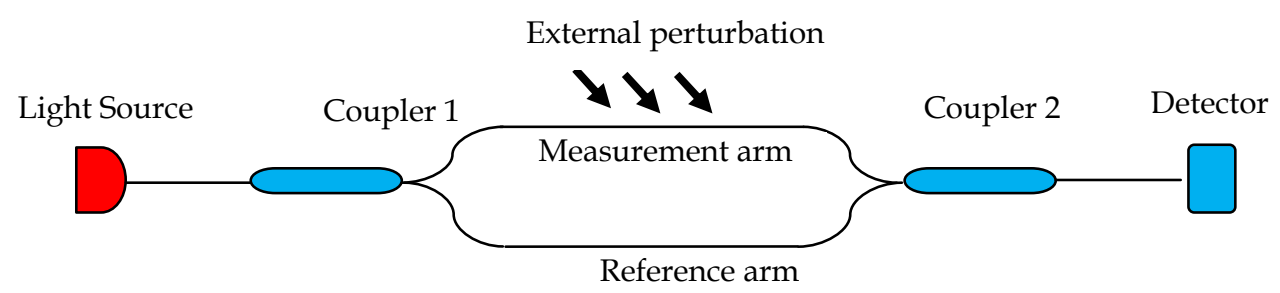

Fig. 7. Mach-Zehnder optical fiber interferometer

large range of possible phenomena (thermal, mechanical, chemical, electromagnetical, etc.).

\section{Sagnac effect}

When two counterpropagative waves propagate in an optical fiber loop in rotation (figure 8), a phase shift appears between the two counterpropagative waves which is proportional to the rotation rate $\Omega(\mathrm{rad} / \mathrm{s})$. This phenomenon is called the Sagnac effect [Sagnac, 1913].

The phase shift is:

$$
\Delta \phi_{S}=\frac{8 \pi R L}{\lambda_{0} c} \Omega
$$

Where :

$L$ is the length of optical fiber in the loop,

$\Omega$ is the rotation rate $(\mathrm{rad} / \mathrm{s})$.

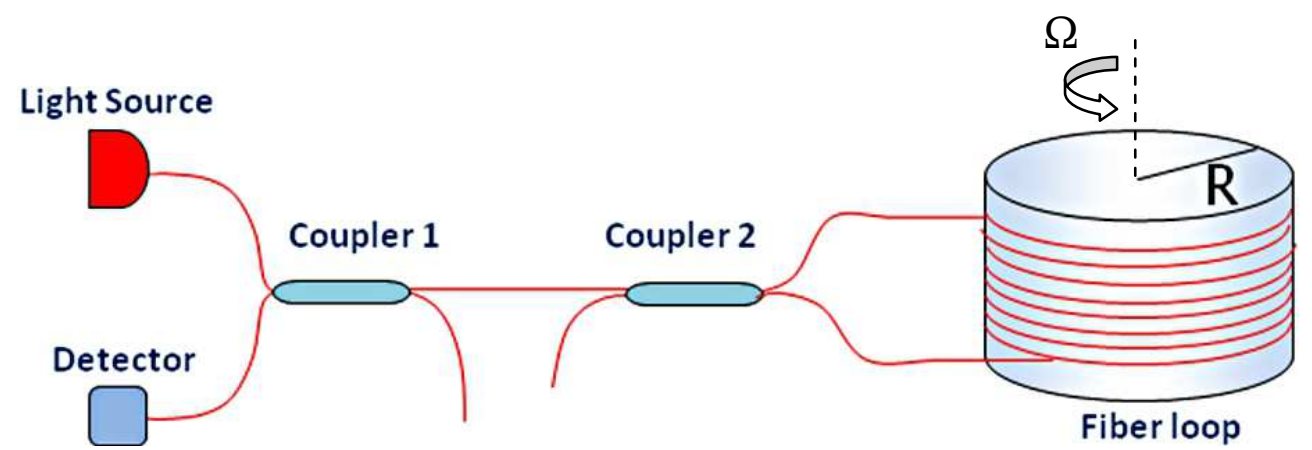

Fig. 8. Sagnac optical fiber interferometer

Interferometers as described in figure 8 allow to measure this phase shift and, therefore, to deduce the rotation rate [Burns et al., 1983][ Zarinetchi et al., 1991].

Generally, this kind of gyroscope is made with monomode polarization maintaining fibers. Moreover, in order to allow unambiguous measurements, the maximum difference of the optical pathway cannot be larger than $\lambda_{0}$. Therefore, in the visible range, a LED whose coherence length is around $3 \mu \mathrm{m}$ is enough to create the needed interferences. 


\subsection{Spectral modulation}

In this case, the external physical phenomenon to be measured modifies the source spectre in its transmission or reflexion.

\section{Bragg grating}

A fiber Bragg grating is an optical fiber where a periodic modulation of the refractive index has been written in its core. Thus refractive index modulation is often photo-written using UV Eximer laser. Because of this index periodicity, several given wavelengths $\lambda_{p}$ will be reflected (Fig.9):

$$
\lambda_{p}=2 n_{e} \Lambda / p
$$

where $\Lambda$ is the grating period, $p$ an integer and $n_{e}$ the effective refractive index of the grating.
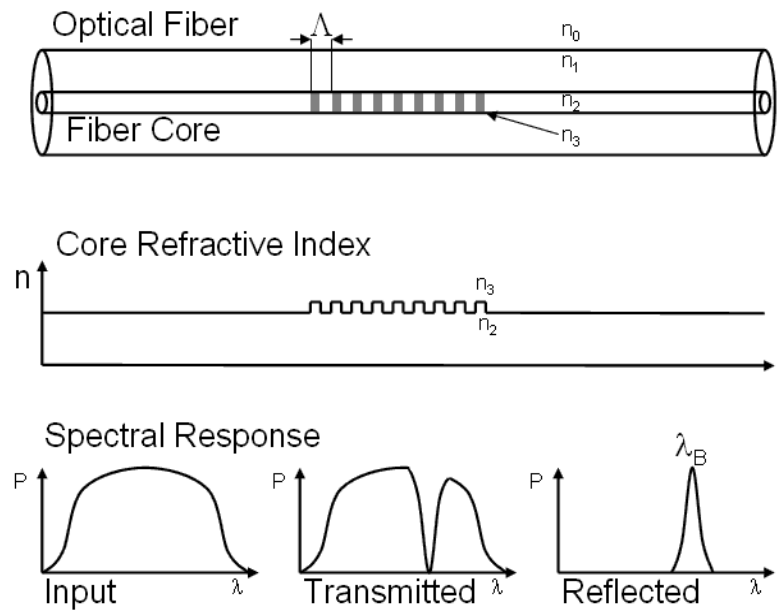

Fig. 9. Fiber Bragg grating principle

Stretching, temperature or pressure may change the reflected wavelength due to a change in the refractive index. To measure this change in the spectrum, a monochromater or a scanning wavelength laser has to be used. These two devices are quite expensive. However the fiber Bragg grating sensors, because of their accuracy, are the more used optical fiber sensors in the industry. They allow the measurement of several physical parameters:

- $\quad$ Stretching and force [Kang et al., 2007 | Ferraro et al., 2002] ;

- $\quad$ Temperature [Zhao et al., 2007 | Zhan et al., 2007 | Hirayama et al., 2000] ;

- $\quad$ Pressure [Takahashi et al., 2000 | Ni et al., 2007] ;

- Two physical parameters together [Hathaway et al., 1999].

The typical sensitivities of fiber Bragg gratings are summarized in table 1 [Lee, 2003].

\begin{tabular}{|l|l|l|l|}
\hline Parameters & stretching & temperature & pressure \\
\hline Sensibility $\Delta \lambda$ & $1 \mathrm{pm} / \mu \mathrm{m}$ & $12 \mathrm{pm} /{ }^{\circ} \mathrm{C}$ & $-0.5 \mathrm{pm} / \mathrm{bar}$ \\
\hline
\end{tabular}

Table 1. The typical sensitivity of fiber Bragg gratings [Lee, 2003]. 
Several Bragg gratings with different periods can be used in the same fiber what allows to achieve a distributed sensor with several measurement localizations.

\section{Fabry-Perot}

Some spliced fibers are used as a Fabry-Perot cavity whose resonance is shifted as a function of the external physical perturbation to measure. The shift can be due to the elasto-optic effect, the thermo-optic effect, the Sagnac effect, etc. It can take place inside the fiber or at the fiber ends.

\section{Multimode optical fiber intrinsic sensor: The principles}

With the multimode optical fiber sensor, the physical phenomenon to be measured modifies the transmitted intensity. An optical fiber is multimode when its normalized frequency is larger than 2.405. Multimode fibers often have a larger core radius (compared to the wavelength) or a higher numerical aperture than a monomode fiber. In this case, several modes defined by different transverse intensity distributions and propagation constants can be guided inside the optical fiber. The transmitted intensity modulation is due to mode couplings between the guided modes and the radiated ones. The mode couplings are the exchanges of energy between modes and the radiated modes are the propagative modes which are not guided inside the fiber but go out in the optical cladding and are generally absorbed by the mechanical jacket. The mode couplings will be due to perturbations of the optical fiber.

\subsection{Micro bending and mode coupling}

The micro bending is a classical perturbation of a multimode fiber. In this case, the fiber is periodically curved (Fig.10). If $z$ is the initial axis of the fiber, the new one is a periodic function $f(z)$. The micro bending may change the local numerical aperture creating losses [Remouche et al., 2007].

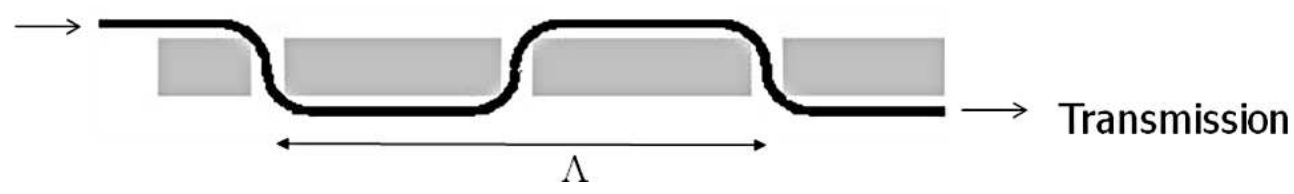

Fig. 10. Micro-bending of a multimode optical fiber

In an optical fiber of refractive index $n_{c}$ in the core and $n_{g}$ in the optical cladding, the guided modes have a propagation constant $\beta_{\mathrm{g}}$ between $2 \pi n_{g} / \lambda_{0}$ and $2 \pi n_{c} / \lambda_{0}$ whereas the propagation constants of the radiated modes $\beta_{\mathrm{r}}$ range between 0 and $2 \pi n_{g} / \lambda_{0}$ (Fig.11). A guided mode of propagation constant $\beta_{\mathrm{g}}$ can be coupled with a radiated mode of propagation constant $\beta_{\mathrm{r}}$ if the geometric perturbation has a period $\Lambda$ such as:

$$
\beta_{g}-\beta_{r}=\frac{2 \pi}{\Lambda}
$$

For a small bending period, the guided modes will be coupled together prior to being coupled with the radiated modes. This method can be used to distribute the source energy 


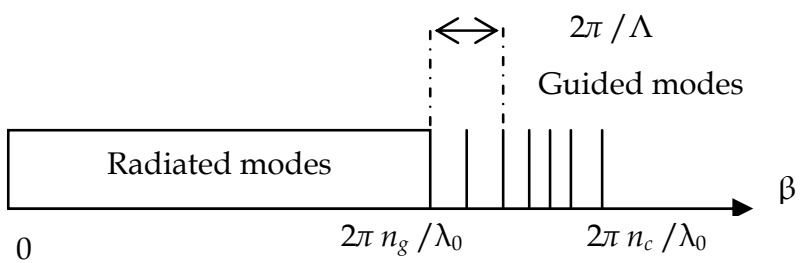

Fig. 11. Propagation constant distribution in an optical fiber: example of two coupled modes

on the entire guided mode of the fiber. In contrary, when the bending is too high, the optical fiber can have inelastic modifications and can be broken. The maximum curvature radius of a classical silica/silica fiber is around $1.5 \mathrm{~mm}$.

The losses can be also explained and modelled by using ray tracing (Fig.12).

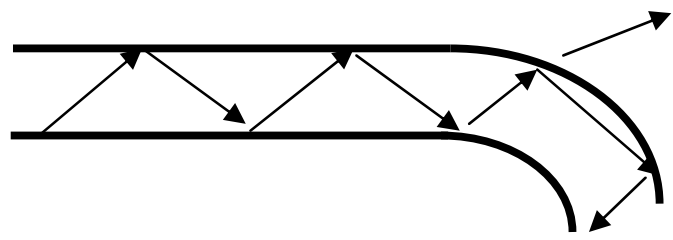

Fig. 12. Ray tracing in a micro bend optical fiber

The physical parameters which can be measured using micro-bending optical fiber sensors are pressure, force [Lagakos et al., 1987], acceleration, temperature [Yadav et al., 2007], Stretching [Luo et al., 1999] and the electromagnetic field [Lagakos et al., 1987].

It is also possible to periodically perturb the optical fiber diameter. The coupling model has been developed by Marcuse [Marcuse, 1991].

\subsection{Spatial mode filtering}

When a multimode fiber is welded to a monomode one a spatial mode filtering is achieved (Fig.13). This principle has been used to realize a stretching sensor [Ribeiro et al., 2004]: the physical parameter to be measured creates mode couplings in the multimode fiber and the monomode fiber allows to filter only the fundamental mode.

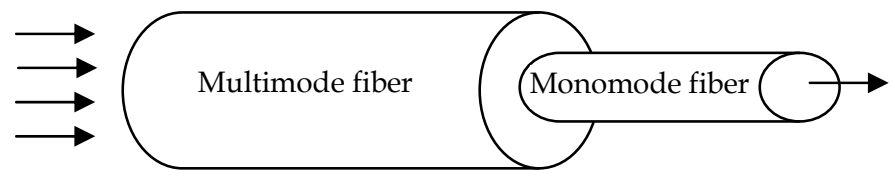

Fig. 13. Spatial mode filtering using welded multimode and monomode fibers

\subsection{Scattering and fluorescence}

The multimode optical fiber sensors are mainly amplitude modulation sensors. Independently of the geometry several others phenomena can achieve an amplitude modulation linked to a physical parameter to be measured: 
- Absorption. It is linked to the imaginary part of the refractive index.

- Rayleigh scattering. It is an elastic scattering: the wavelength is conserved. The light is spatially dispersed because of optical fiber defects smaller than the wavelength, what makes decrease the transmitted intensity. The Rayleigh scattering can be measured with a spatial localization using an OTDR (Optical Time-Domain Reflectometer).

- Raman scattering. It is a non linear inelastic scattering where electromagnetic and acoustic energies are exchange. Stokes rays with frequency smaller than the source and anti-Stokes rays with frequency higher than the source appear. Only the anti-Stokes ray depends of the temperature, what has interest for metrology.

- Fluorescence. Molecules are exited for example by absorbing a radiation or a particle and then emit a photon when they come back to their fundamental state. Fluorescence can be linear or not, elastic or not. The typical time between absorption and emission is an important parameter. UV and gamma optical fiber sensors are achieved using this principle, biological sensors also.

- Brillouin scattering. It is a non linear scattering: a frequency shift takes place due to interaction between the light wave and acoustic waves (Stokes ray). The frequency shift is in the range of $10 \mathrm{GHz}$ and typically depends of temperature and stress in the fiber. A large range of optical sensors have been achieved using this phenomenon measuring spectrum or absorption. Dynamic and distributed sensors can be done using the time modulation of the acoustic wave.

\subsection{Transimpedance circuit}

In an amplitude modulation optical fiber sensor the transmission can be measured using a simple photodiode. In this case, a transimpedance circuit (figure 14) allows minimizing the noise level. The photo current out of the diode is converted into a stable output voltage. Since there is no voltage across the diode, there is no black current and the diode response is linear. The resistance $R_{f}$ allows having an adjustable amplification:

$$
V_{\text {out }}=R_{f} I
$$

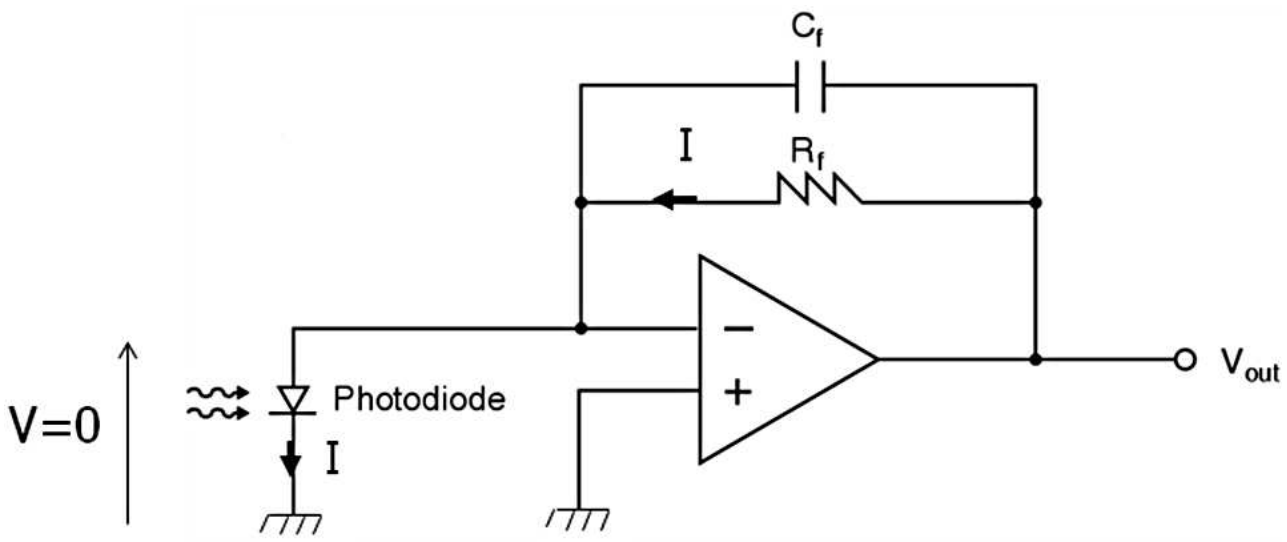

Fig. 14. Transimpedance circuit 


\section{Example of intrinsic optical fiber sensing engineering and examples}

Figure 15 represents the ratio of scientific papers concerning the various physical parameters to be measured with optical fiber sensors [Lee, 2003]. The more studied parameters are stretching, temperature and pressure. The used technologies for measurements using optical fiber sensors are shown in figure 16.

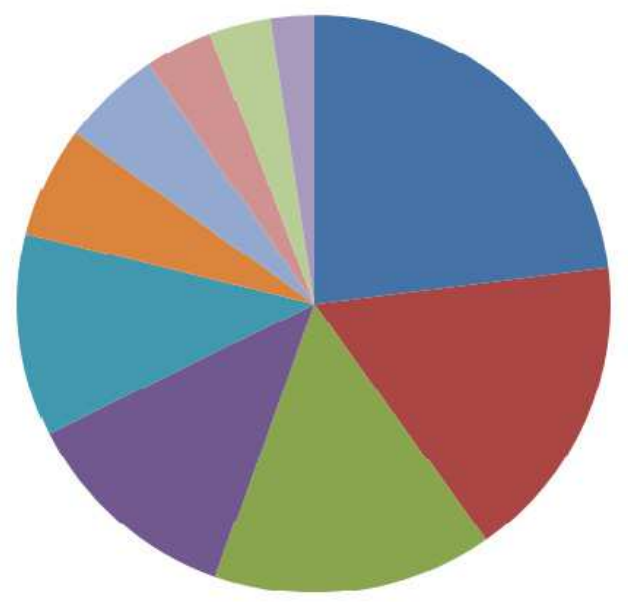

$$
\begin{aligned}
& \text { - Stretching } \\
& \text { - Temperature } \\
& \text { - Pressure/Acoustic } \\
& \text { nCurrent/Voltage } \\
& \text {-Chemistry/gas } \\
& \text { - Rotation } \\
& \text { - Vibration/Acceleration (5.5\%) } \\
& \text { - Bending/Torsion } \\
& \text { Displacement } \\
& \text { - Bio }
\end{aligned}
$$

Fig. 15. Ratio of scientific papers concerning the different physical parameters to measure [Lee, 2003]

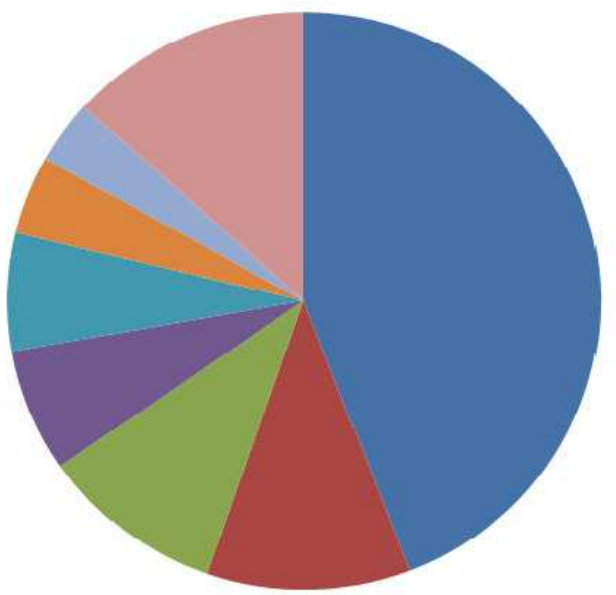

Grating fiber

Interferometer

-Scattering/Reflex ion

-Faraday effect

-Fluorescence

Gyroscopes

Low coherent interferometer $(3.5 \%)$

Others

Fig. 16. used technologies for measurements using optical fiber sensors [Lee, 2003] 


\section{Bragg grating fiber}

The Bragg grating fiber is the more studied technology [Lee, 2003].

Bragg grating optical fiber sensors are used to measure temperature, pressure, stretching, force, acceleration, etc. [Ferdinand et al., 1997]. Inclinometers have also been achieved.

\section{Force, vibration measurements and shock detection}

Force, vibration and shock detection measurements can be carried out using micro bends in multimode fibers or using polarization modulation out of monomode fibers. Microphones, hydrophones (US Navy), seismometers, vibrometers have been achieved. Large dynamic can be considered. These technologies allow achieving long lifetime and low cost sensors that could be applied in the automotive applications.

Such measurements can also be done using distributed Bragg grating fibers or Brillouin sensor. For example, Bragg grating fibers have been used for railway monitoring.

\section{Torque measurement}

Torque measurement can be achieved using polarization study out of a twist monomode optical fiber. The measurement is indirect; the applied torque will be linked to the measured twist.

\section{Rotation rate measurement}

The optical fiber gyroscopes are one of the more accurate gyroscopes. Rotation rates smaller than $0.1^{\circ} / \mathrm{h}$ could be detected using maintaining polarization fibres. These quite expansive gyroscopes (several 10k\$) are used for example for military navigation applications. These sensors have no moving mechanical parts and are resistant to electromagnetic field. Several configurations have been proposed: open loop interferometer, resonant ring, Brillouin gyroscope, etc. Cheaper multimode optical fiber gyroscopes have also been proposed for rate-grade applications [Medjadba et al., 2011] when classical micromechanical systems cannot be used.

\section{Temperature measurement}

The main effect that will be used is the thermo-optic effect, which is the change of refractive index of a material due to the temperature. This phenomenon is characterized by the thermo-optic coefficient $a$ :

$$
n=n_{T_{o}}+\alpha\left(T-T_{o}\right)
$$

The thermo-optic coefficient of silica is around $1.45 .10^{-5} \mathrm{~K}^{-1}$. This change of refractive index may change the reflected wavelength in a Bragg grating fiber sensor, the transmission in an amplitude modulation sensor, the phase shift in a Mach-Zhender sensor, etc.

In the amplitude modulation sensor, to increase the change of numerical aperture due to temperature, different materials are used in core and cladding. Typically the core will be in silica and a polymer with a negative thermo-optic coefficient will be used. The fiber with polymer can generally not be used for temperature larger than $50^{\circ} \mathrm{C}$, whereas silica fiber can be used until $800^{\circ} \mathrm{C}$. 
The dependence of the Brillouin scattering on the temperature is also used to achieve temperature sensors. Another principle that could be used is the change of Young modulus as a function of the temperature in a perturbed plastic optical fiber.

\section{Gas and pollution detection}

Gas and pollution detection are possible using absorption or fluorescence in amplitude modulation sensor. Refractive index change can also be obtained in some materials. Hydrogen is absorbed by palladium coating for example, or Rayleigh scattering due to pollution can increase absorption in hollow photonic crystal fiber.

\section{Shape and stretching measurement}

Shape and stretching can be measured by observing bending change or Bragg grating period elongation. Optical stress gauge can be achieved. Distributed sensors have been used in geni-civil applications as strain highway bridge monitoring and mine security control.

\section{Magneto optic current sensor}

Polarization modulation sensors or Sagnac interferometer could be used to measure currents due to the induced magnetic field and the Faraday effect. This measure can be carried out without contact and electromagnetic perturbation of the studied system.

\section{Defence applications}

The possibility to use optical fiber sensors in explosive environments or in an area where high electric or magnetic field take place, are often researched advantages for military applications. No moving mechanical part is also useful when a sensor is submitted to high accelerations.

One of the more used optical fiber sensor in the defence application is the optical gyroscope for navigation mainly because is high accuracy.

\section{Ecology and biomedical applications}

The optical fibers are used for biological studies: grow of cells, kinetic of binding reactions, chemical evolution monitoring, etc. using refractometry, interferometer, amplitude modulation, etc. The biological samples can be located at the fiber end, as an optical cladding or in the hollow core of a photonic crystal fiber. Integrated biochip can be imagined. Gas detection and concentration measurements can be done. The distributed in-situ temperature and strain measurements have also been studied for medical applications, inside the body.

\section{Prospectives: Sensing with photonic crystal fibers and plasmonic sensors}

New optical fiber structures and materials, but also the improvements of the micro and nano fabrication processes allow the development of new families of optical fiber sensors. These new sensors are often still in development and need additional time before to be mature for industrial applications. However, nowadays a many patents are deposed in order to protect these future technologies.

\subsection{Micro structured fibers}

The classical optical fibers are made of bulk materials, but now it is possible to micro structure the core or the optical cladding of the fiber. These new fibers can be decomposed 
into two families: the photonic crystal fiber and effective index optical fiber. Because of their guiding principles these fibers are highly sensitive to external physical parameters (temperature, pressure, bending, etc.). They have been used for example to achieve biochemical sensors [Rindorf et al., 2006], gas detection [Hoo et al., 2003], etc.

\section{The photonic crystal fiber}

These fibers (figure 17b) have a hollow core and the guidance properties are due to a photonic band gap in the periodically structured optical cladding [Bjarklev, 2003]. A photonic band gap is a spectral domain for which the light cannot propagate in the medium: it appears in structures where the optical properties are periodic with a period that can be compared to the wavelength and where the refractive index contrast is higher than 2 . The periodic structure is generally done by using air and another material. The hollow core allows not only a low dispersive propagation but also the insertion of gas to study inside the fiber. Liquids can also insert in the hollow core, as for example for the photonic liquid crystal fiber.

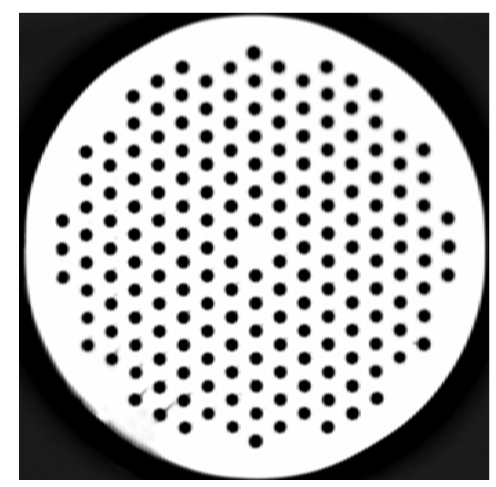

(a)

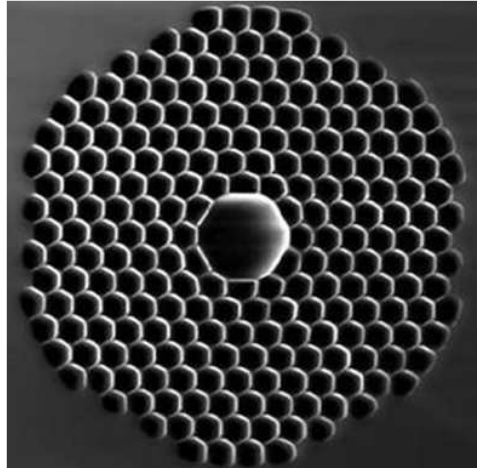

(b)

Fig. 17. Micro structured optical fiber: (a) Effective index fiber, (b) photonic crystal fiber

\section{Effective index optical fiber}

The core of these optical fibers is full and the cladding is structured at a scale smaller than the wavelength in order to create an effective medium with an effective refractive index smaller than the one of the core (figure 17a).

Temperature and strain sensors, gas detectors and refractometers have yet been experimented using these new fibers. The polarization maintaining photonic crystal fiber and the large mode area photonic crystal fiber, both monomode, are specifically studied.

\subsection{Plasmonic sensors}

A plasmon is a surface electromagnetic wave linked to a surface current. These waves propagate along a metallic surface and are evanescent in other directions. Because of the metallic complex refractive index, the plasmons are generally highly absorbed allowing amplitude modulation sensors. Plasmon resonances can occur in thin metal layers (several ten nanometers thickness for visible range resonances). These resonances are very sensitive 
to the environment (refractive index), therefore sensors have been achieved. In this case we talk about Surface Plasmon Resonance optical fiber sensor.

Figure 18 is an example of plasmonic optical fiber sensor for hydrogen detection [Perrotton et al., 2011]. Locally, the optical cladding has been replaced by a thin $10 \mathrm{~nm}$ layer of palladium. The adsorption of hydrogen by the metallic palladium layer changes its complex refractive index and therefore its absorption and resonance spectrum. Ratio of Hydrogen smaller than $4 \%$ has to be detected. It's an example of optical sensors used in an explosive environment in order to minimize risks. The plasmon excitation can also be done on a thin layer at the end of the fiber. In this case, the detection takes place in reflexion.

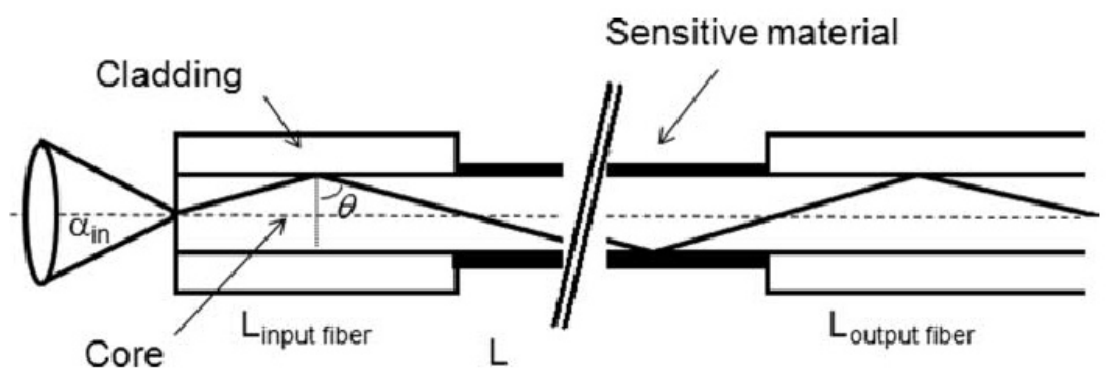

Fig. 18. Plasmonic optical fiber sensor for hydrogen detection. In black: palladium layer

This family of new sensors are sometime classified as evanescent field sensors because a plasmon is a particular case of evanescent waves. This wave is used as a very sensitive and localized probe to sense a system.

\section{Conclusion}

We have seen that many simple physics principles can be efficiently used for intrinsic optical fiber sensing through an appropriate engineering. With the new potential open by photonics crystal fibers and integrated optics probably many others applications in optical fiber sensing of these physics principles are going to be discovered in the years to come.

The main inconvenient of intrinsic optical fiber sensing is to sense "everything" at the same time. This inconvenient was progressively overcome.

It is possible to say, now, that optical fiber sensing is a mature technology able to solve many measurements and control problems, and not only in the laboratory.

The optical fiber sensing industry is just emerging. Its principal burden is to deal with the lack of trained engineers and technician in this field. What make that even if the basic knowledge on optical fiber sensing is now abundantly available in literature, know-how to proceed is scarcely diffused. An educational effort is necessary, but it will provide positive results only with long delay.

Anyway the engineers and scientists from many fields have to be aware of the existing capacities of optical fiber, to be able to consider them for optimizing their process or devices by trying to find an assistance among the small number of specialized laboratories. 
Since innovation is going to be the main motor of the future economy, optical fiber sensing can bring many progress for innovative products, even if it is difficult presently to associate experts in optical fiber sensing to projects because these experts are not enough numerous.

So optical fiber sensing has the potential, in the industrial world, to transform its statute by evolution from a niche technology to a basic enabling industrial growth opportunity that can become strategic in : transportation, biomedical, defense, ecology, energy, etc.

Optical fiber sensing offers a tool to fabricate products more secure, less polluting or poisonous, saving energy and rare materials.

Optical fiber sensing belong at the same time to the past by being related for instance to the Nobel Prize of M. KAO for optical fiber and to the future because it allows to imagine and to realize creative products implementing new functions with many advantages.

In this chapter we have tried to summarize the state of the art in optical fiber sensing. We hope that this chapter will rise the interest of engineers and scientists not aware of the strong opening to innovations of optical fiber sensing, and that they will follow by trying to learn more specifically from optical fiber sensing laboratories that deserve assistances of any kind to contribute to the rewarding accomplishments that, we hope, are going to take place in the future through classical and disruptive optical fiber sensing technologies.

\section{Acknowledgment}

The authors thank Ayoub Ckakari, Pierre Pfeiffer, Nicolas Javahiraly, Sylvain Fischer, Bruno Serio, Francis Georges, Anthony Bichler, Cédric Perrotton, Hocine Medjadba and Mustafa Remouche that work with us on optical fiber sensors and share their experience.

\section{References}

A. Bjarklev, A. S. Bjarklev, J. Broeng, Photonic crystal fibers, Springer 2003.

W. K. Burns, R. P. Moeller, C. A. Villaruel, and M. Abebe, Fiber-optic gyroscope with polarization-holding fiber, Opt. Lett., Vol. 8, pp. 540-542, 1983.

P. Ferdinand, S. Magne, V. Dewynter-Marty, C. Martinez, S. Rougeault, and M. Bugaud, Applications of Bragg grating sensors in Europe, in Optical Fiber Sensors, OSA Technical Digest Series, 1997

P. Ferraro, G. De Natale, On the possible use of optical fiber Bragg gratings as strain sensors for geo dynamical monitoring, Optics and Lasers in Engineering, 37, 2002, pp. 115-130

M. Ferretti, Capteurs à fibres optiques, Techniques de l'ingénieur, traité Mesures et Contrôles, R415, 1996

S.K. Ghorai, D. Kumar, B.K. Hura, Strain measurement in a Mach-Zehnder fiber interferometer using genetic algorithm, Sensors and Actuators A, 122, 2005, pp. 215-221

K.T.V Grattan, T. Sun, Fiber optic sensor technology: an overview, Sensors and Actuators, 82, pp. 40-61, 2000

M.W. Hathaway, N.E. Fisher, D.J. Webb, C.N. Pannell, D.A. Jackson, L.R. Gavrilov, J.W.

N. Hirayama, Y. Sano, Fiber Bragg grating temperature sensor for practical use, ISA Transactions, 39, pp. 169-173, 2000 
Y. L. Hoo, W. Jin, C. Shi, H. L. Ho, D. N. Wang, and S. C. Ruan, Design and Modeling of a Photonic Crystal Fiber Gas Sensor, Appl. Opt. 42, 3509-3515, 2003

L. Kang, D. Kim, J. Han, Estimation of dynamic structural displacements using fiber Bragg grating strain sensors, Journal of Sound and Vibration, 305, 2007, pp. 534-542

N. Lagakos, J.H. Cole, J.A. Bucaro, Microbend fiber-optic sensor, Applied Optics, Vol. 26, N¹1, pp. 2171-2180, 1987

B. Lee, Review of the present status of optical fiber sensors, Optical Fiber technology, 9, pp. 57-79, 2003

F. Luo, J. Liu, T.F. Morse, A fiber optic microbend sensor for distributed sensing application in the structural strain monitoring, Sensors \& Actuators, 75, pp. 41-44, 1999

D. Marcuse, Theory of dielectric optical waveguides, Edition : Academic Press, Second Edition, 1991

H. Medjadba, S. Lecler, L. M. Simohamed, J. Fontaine, R. Kiefer , An optimal open-loop multimode fiber gyroscope for rate-grade performance applications, Optical Fiber Technology, 2011

X. Ni, Y. Zhao, J. Yang, Research of a novel fiber Bragg grating underwater acoustic sensor, Sensors and Actuators A, 138, 2007, pp. 76-80

C. Perrotton, M. Slaman, N. Javahiraly, H. Schreuders, B Dam, Wavelength response of a surface plasmon resonance palladium-coated optical fiber sensor for hydrogen detection, Optical Engineering 50(1), 2011

K. Peters , Polymer optical fiber sensors - a review, Smart Mater. Struct. 20 013002, 2011.

M. Remouche, R. Mokdad, M. Lahrashe, A. Chakari and P. Meyrueis, Intrinsic optical fiber temperature sensor operating by modulation of the local numerical aperture, Opt. Eng. 46, 024401, 2007

R.M. Ribeiro, M.M. Werneck, An intrinsic graded-index multimode optical fibre strain-gauge, Sensors and Actuators A, 111, pp. 210-215, 2004

L. Rindorf, J. B. Jensen, M. Dufva, L. H. Pedersen, P. E. Høiby, and O. Bang, Photonic crystal fiber long-period gratings for biochemical sensing, Opt. Express 14, 8224-8231, 2006

G. Sagnac, L'éther lumineux démontré par l'effet du vent relatif d'éther dans un interféromètre en rotation uniforme, Compte-rendu de l'Académie de Sciences, Vol. 157, pp. 708-710, 1913.

N. Takahashi, K. Yoshimura, S. Takahashi, K. Imamura, Development of an optical fiber hydrophone with fiber Bragg grating, Ultrasonics, 38, 2000, pp. 581-585

D. Yadav, A.K. Nadir, Transformer temperature measurement using optical fiber based microbend sensor, Sensors \& Transducers Journal, Vol. 84, N¹0, pp. 1651-1659, 2007

L-B Yuan, L-M Zhou, J-S Wu, Fiber optic temperature sensor with duplex Michelson interferometric technique, Sensors and Actuators, 86, pp. 2-7, 2000

F. Zarinetchi, S. P. Smith, S. Ezekiel, Stimulated Brillouin fiber-optic laser gyroscope, Opt. Lett., Vol. 16, pp. 229-231, 1991

Y. Zhan, S. Xue, Q. Yang, S. Xiang, H. He, R. Zhu, A novel fiber Bragg grating hightemperature sensor, Opt. Int. J. Light Electron. Opt, 2007

H., L. Zhang, I. Bennion, Combined ultrasound and temperature sensor using a fibre Bragg grating, Optics Communications, 171, 1999, pp. 225-231 
C. Zhao, M.S. Demokan, W. Jin, L. Xiao, A cheap and practical FBG temperature sensor utilizing a long-period grating in a photonic crystal fiber, Optics Communications, 276, pp. 242245, 2007 


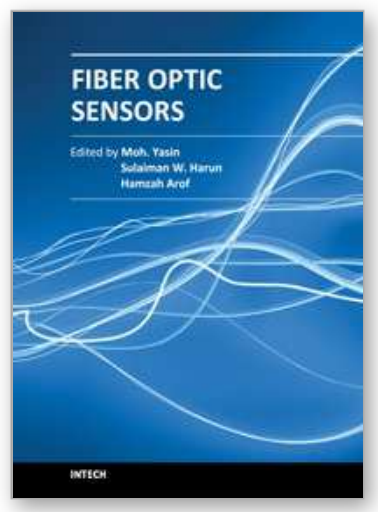

\author{
Fiber Optic Sensors \\ Edited by Dr Moh. Yasin
}

ISBN 978-953-307-922-6

Hard cover, 518 pages

Publisher InTech

Published online 22, February, 2012

Published in print edition February, 2012

This book presents a comprehensive account of recent advances and researches in fiber optic sensor technology. It consists of 21 chapters encompassing the recent progress in the subject, basic principles of various sensor types, their applications in structural health monitoring and the measurement of various physical, chemical and biological parameters. It also highlights the development of fiber optic sensors, their applications by providing various new methods for sensing and systems, and describing recent developments in fiber Bragg grating, tapered optical fiber, polymer optical fiber, long period fiber grating, reflectometry and interefometry based sensors. Edited by three scientists with a wide knowledge of the field and the community, the book brings together leading academics and practitioners in a comprehensive and incisive treatment of the subject. This is an essential reference for researchers working and teaching in optical fiber sensor technology, and for industrial users who need to be aware of current developments and new areas in optical fiber sensor devices.

\title{
How to reference
}

In order to correctly reference this scholarly work, feel free to copy and paste the following:

Sylvain Lecler and Patrick Meyrueis (2012). Intrinsic Optical Fiber Sensor, Fiber Optic Sensors, Dr Moh. Yasin (Ed.), ISBN: 978-953-307-922-6, InTech, Available from: http://www.intechopen.com/books/fiber-opticsensors/intrinsic-optical-fiber-sensor

\section{INTECH}

open science | open minds

\section{InTech Europe}

University Campus STeP Ri

Slavka Krautzeka 83/A

51000 Rijeka, Croatia

Phone: +385 (51) 770447

Fax: +385 (51) 686166

www.intechopen.com

\section{InTech China}

Unit 405, Office Block, Hotel Equatorial Shanghai

No.65, Yan An Road (West), Shanghai, 200040, China

中国上海市延安西路65号上海国际贵都大饭店办公楼 405 单元

Phone: +86-21-62489820

Fax: $+86-21-62489821$ 
(C) 2012 The Author(s). Licensee IntechOpen. This is an open access article distributed under the terms of the Creative Commons Attribution 3.0 License, which permits unrestricted use, distribution, and reproduction in any medium, provided the original work is properly cited. 